\title{
Lois du silence, lois de la parole ou comment penser avec Foucault les politiques du dire
}

\author{
Catherine Mavrikakis
}

This text considers the ethical and political, unconscious and institutional stakes of the act of silence-breaking. What motivates an individual to declare that which has been kept hidden? Does breaking silence in order to accuse (oneself?) inevitably reinscribe a mode of power? What new silences are produced through acts of silence-breaking? Can a confession ever be subversive or revolutionary? In order to reflect on the way that neither silence nor speech can exist as an absolute sign, Catherine Mavrikakis intersperses a series of speech acts unveiling personal and family secrets with considerations of the different effects of confessions in the contexts of criminal jurisprudence, Presidential image-making, state apologies, talk-show traumas, and educational institutions.

« Nous n'avons parlé que lorsque nous n'eussions pu nous taire sans crime ». Pascal

C'est à une théorie du secret et de l'aveu qu'une réflexion sur la loi, surtout celle du silence et de la parole me mènera ici.

$C^{\prime}$ est sur les enjeux inconscients, institutionnels, politiques et éthiques de cette trahison du silence que je voudrais réfléchir. Celle qui prend la parole, qui rompt le silence pour (se) dénoncer, ( $s^{\prime}$ )accuser s'inscrit-elle par avance dans un système de pouvoir intrinsèque à toute parole ? Peutil y avoir des aveux subversifs ou encore révolutionnaires ? Quelle performativité et quelle politique du dire s'inscrivent dans le passage du silence à la parole et particulièrement dans la prise de parole par les femmes?

De cette performativité du silence et de la parole, je jouerai ici en mettant en scène des situations de rupture de silence. Je voudrais ainsi réfléchir sur les liens qui se tissent entre loi du silence, femmes et pouvoir dans nos sociétés.

Les bris de silence que je mets en scène ici, bris d'intimité, de confidentialité veulent dessiner un espace où la loi, celle du silence, aussi bien que celle de la parole, est mise en accusation, mais peut aussi se retourner contre moi et $\mathrm{m}^{\prime}$ accuser $\mathrm{du}$ pire. 


\section{Pour qui se tait-on ? Pour qui parle-t-on?}

\section{L'aveu numéro un. Le premier d'une longue chaîne}

Je, soussignée, Catherine Marie Marchand Mavrikakis, née le 7 janvier 1961 à Chicago (U.S.A), domiciliée au 475 Prince-Arthur, app.4, à Montréal (Québec), professeure agrégée à l'Université Concordia, avoue pour la première fois, de façon publique et écrite, avoir eu des relations incestueuses avec mon demi-frère Patrice Risi, né le 14 mai 1947 à Paris (France) et mort le 17 décembre 1996 à Ostende (Belgique) durant ma toute petite enfance.

Je passe aux aveux, je brise la chape de plomb du tu.

Je mets en scène ma sortie du silence.

Je ne dis rien. Ou presque. Ou juste assez pour passer pour hystérique, folle ou dans la provocation.

Théâtralité d'un traumatisme qui ne se dirait pas, qui se mettrait en scène seulement, qui nous dirait seulement qu'il est là, qu'il gît.

Ou plutôt qui ne pourrait se dire pleinement que devant ce destinataire, complice, bourreau, ma victime aussi ; traumatisme qui prit place seulement, dirais-je, pour et devant les beaux yeux de ma mère.

Ma mère : à qui, bien sûr, je n'adresse pas mes aveux. Pas directement. C'est déjà fait.

Briser le silence, pour justement ne pas dire. En finir néanmoins avec un certain fantasme de dire.

Parler pour faire silence. Faire de mon aveu, un silence. Et créer le silence chez les autres. Ou encore un malaise. Alors pourquoi la comédie d'en finir avec un certain silence ? Pourquoi cela dans Tessera ? Parce que c'est une des revues que ma famille, ma mère ne lit certainement pas, dirai-je non sans un petit brin de malice. C'est dans Tessera que je peux jouer avec mon silence.

Le silence, comme la parole, n'existe pas comme signe absolu. Il n'existe que dans un contexte, et est toujours lié à un destinataire. On se tait pour et en face de quelqu'un.

Quels aveux n'arrive-t-on pas à faire, dans le creux du divan ?

Mais entre passer aux aveux en psychanalyse ou dans une revue féministe, il y a un monde et plusieurs niveaux de silence. Des silences stratégiques et des prises de parole tactiques.

Briser le silence en analyse, devant celui ou celle qui ne pourra que le garder, sous le sceau du secret professionnel n'est pas la même chose que de le faire devant un ami, ou dans un écrit. L'aveu dessine une stratégie du destinataire, et construit souvent celui-ci comme complice d'un certain silence ou du contenu de la parole. Tessera est-elle ma complice dans le crime de dire? 
Entre le silence comme absolu et le fantasme d'une parole universellement communicable, que l'on pourrait crier sur tous les toits, il y a tous les aveux, toujours répétés et tous les diverses mises en scène de silences.

Je vais tout avouer, dix mille fois, mais jamais je n'aurai le courage de briser le silence, je ne parviendrai pas à lui enlever pour de bon son hymen. Parce que je n'arriverai pas à le dire à celui ou celle qui tiendrait lieu de ce "sur tous les toits ». Si le silence peut être absolu, en sortir, le briser est toujours une stratégie de son dévoilement, une impossibilité à le défaire dans sa totalité.

Si l'on en croit Michel Foucault, l'État moderne se fonde sur la nécessité pour le sujet d'en passer par le processus de l'aveu, de briser le silence, afin de permettre aux instances institutionnelles, de se fonder dans une alliance du psychiatrique et du judiciaire, du personnel et du juridicoinstitutionnel. L'État, en d'autres termes, force les individus à se reconnaître comme sujets dans les aveux qu'ils font.

L'histoire de Pierre Rivière est significative à ce sujet. Pierre Rivière tua sa mère, sa sœur et son frère afin de défendre son père. Étant donné ce crime incompréhensible, le juge d'instruction demanda à Pierre Rivière d'écrire son meurtre.

Le cas de Pierre Rivière montre qu'à partir du dix-neuvième siècle, le juridique étatique ne demande pas seulement à l'individu d'avouer ses fautes, mais de briser tous les silences, de scruter l'intériorité de la conscience afin de la faire sortir de son retrait. C'est seulement après cette totale confession que la punition peut s'abattre, et l'on pourrait dire alors que l'aveu fait déjà partie de la punition. Le passage du silence à la parole consolide le pouvoir et force l'individu à se soumettre aux instances politiques et à juger de ses propres actions et de son pouvoir.

On peut penser à toute l'économie politique de l'aveu dans l'affaire Clinton où le Président des États-Unis en arrive à avouer, après maintes mises en scène de dénégation, avoir eu des relations sexuelles avec Monica Lewinski. L'aveu de Clinton fera le tour du monde, sans que le Président ait à démissionner. On pourrait même dire que Clinton est passé maître dans la compréhension et la stratégie de l'aveu comme force politique : au début de son mandat, il avoua en bloc avoir fumé de la drogue et avoir eu des maîtresses.

La Présidence américaine s'instaure sur la régulation de l'aveu et c'est même presque un rite initiatique pour chaque candidat à l'investiture présidentielle d'avouer une faute de jeunesse, sur laquelle il n'est pas de bon ton de garder le silence. 
Même l'État français a dû reconnaître ses torts dans sa complicité avec les nazis durant la Seconde Guerre mondiale. Lorsque Jacques Chirac prend le pouvoir, l'une de ses premières actions est de parler de la responsabilité de la France dans une rafle célébre et tragique des Juifs à Paris, responsabilité que l'ancien Président, François Mitterand, avait toujours refusée. Chirac brise le silence de l'État dans cette affaire, non pas simplement parce qu'il veut réparer un tort, mais aussi pour mieux consolider son pouvoir, qui désormais, ne peut en passer que par une stratégie de l'aveu et une mise en scène de sortie du silence.

Le dictateur reste pour nous celui qui n'avoue pas ses torts, qui ne se soumet pas à cette stratégie du dire. Pinochet, Klaus Barbie sont d'autant plus des monstres qu'ils n'ont pas encore envie d'avouer. L'aveu devient une garantie d'un pouvoir juste.

Le pouvoir ici et maintenant doit être pensé dans la capacité des hommes d'État, ou des instances politiques, à avouer publiquement leurs fautes. Or, le problème du silence, c'est que pour se dire, il doit non seulement dire sa présence, mais il doit aussi se donner un contenu en arrivant dans la parole.

On peut se taire, mais on se tait toujours sur quelque chose. On ne peut simplement dire que l'on se tait, on doit dire sur quoi, et même, quand on ne le dit pas, on dit un peu. On joue avec le silence, perversement. Le silence en politique n'est plus qu'une mise en scène d'un caché que l'on dévoile stratégiquement et qui donne le pouvoir à celui qui a le courage de parler. Or, ce pouvoir conféré à celui ou celle qui parle est peut-être un piège.

Dans L'Inceste, l'écrivaine Christine Angot raconte des scènes d'inceste avec son père :

Il a fait des courses. Il est nu. On ne sort quasiment pas de cette maison d'Isère. Mais nous faisons des balades, il aime beaucoup la nature, il aime beaucoup le calme, il aime se promener dans la montagne et dans les chemins. Quand on rencontre quelqu'un, il dit bonjour clairement. C'est poli. Ça se fait. Il l'a fait. Il faut que je le fasse aussi. Il faut que je sois polie. Il met des clémentines sur son sexe pour que je les mange. C'est dégoûtant, dégoûtant dégoûtant dégoûtant. (206)

Les aveux d'Angot, ses cris sont vus par elle-même comme une impossible parole. Ils sont en quelque sorte d'avance récupérés par les institutions de l'aveu. Elle écrit : 
Ce livre va être pris comme un témoignage sur le sabotage de la vie des femmes. Les associations qui luttent contre l'inceste vont se l'arracher. Même mes livres sont sabotés. Prendre un livre comme une merde de témoignage ce sera du sabotage, mais vous le ferez. Cela bousille la vie d'une femme, cela bousille la vie d'un écrivain, mais ce n'est pas grave comme on dit. (197)

\section{La série des aveux}

Et si je brisais le silence, le mien et celui parfois à demi brisé des autres ?

Si je passais aux aveux, maintenant que j'ai mis en scène l'aveu du pire? $\mathrm{Si}$ je me mettais à tout dévoiler ce que je n'ai pu dire ou ce que j'ai dû taire. Je ne suis tenue à aucun silence ou encore secret professionnel. Je peux parler sans problème. Mais la problématique du dire est d'avance prise dans un système de codes. Puis-je parler ici ? Le dire est toujours lié au juridique, à la loi. Atteinte à la vie privée, à l'image. Briser le silence, mais juste le silence sur soi, et dans certaines circonstances, dans certains lieux, dans la cure psychanalytique, dans les aveux, dans l'intimité, ou encore devant tout le peuple américain. Interdiction de rompre le silence sur les autres. "Seuls les noms ont été changés, dans le but de préserver ...» La protection du privé et les limites entre le privé et le public sont liées à la loi juridique du silence. Un avis d'une cour, par exemple, peut forcer un individu à briser un silence, que l'ordre social et même la loi, lui interdisent de rompre afin de ne pas porter préjudice à autrui. Le silence est complètement géré par l'ordre institutionnel. Le « je ne parlerai qu'en présence d'un avocat » montre assez ce qu'il en est de l'appareil de gestion du silence et de la parole dans nos démocraties. Le silence est un droit, parfois même un devoir, mais l'État et le juridique mettent en place un système de bris de silences, de codes du dire et du tu. Dans la plupart des systèmes juridiques, ce que l'on dit contre soi-même constitue une preuve. Comme l'explique Foucault :

Le droit britannique qui, interdit le témoignage contre soi-même, est une exception. Mais dans la majorité des autres systèmes, à partir du moment où quelqu'un dit quelque chose qui lui porte préjudice, cette chose ne peut être que vraie. Cela constitue un postulat... Notre système juridique accorde une telle valeur de preuve à l'aveu qu'il devient difficile de le rectifier ou de le nier après coup. S'il est vrai que l'extorsion sauvage de l'aveu est une pratique policière habituelle et que la justice, en principe l'ignore, en feignant de fermer les yeux sur 
elle, il est aussi vrai que, en accordant un tel privilège à la confession, le système judiciaire est un peu complice de cette pratique policière qui consiste à l'arracher à tout prix. (tome 2,810 )

Ce qu'il en est du silence et de la parole constitue en fait toujours une pratique de l'aveu, une politique de la culpabilité ou de la dénonciation, par laquelle le sujet s'inscrit ou se réinscrit dans le système social.

L'aveu de la faute, ou encore l'aveu de quelque chose qui pourrait être constitué comme preuve contre le sujet, devient donc en dernière instance la vérité. Et comme l'explique Foucault, il est très difficile de revenir juridiquement sur ses aveux. Tout se passe comme si le passage difficile du silence à la parole tenait lieu de vraie parole. Plus j'ai du mal à briser le silence, plus ma parole risque d'être vue comme vraie. La parole devrait trouver sa caution, son authenticité dans la capacité du sujet à briser le silence. Je donne à ma parole toute sa profondeur du moment qu'il m'est difficile, voire impossible de sortir du silence. De là, dans nos sociétés, toute une perversion du dire, une création du silence pour donner à la parole une force performative qu'elle n'aurait pas sans son poids construit de silence. On assiste alors à une surenchère de la valeur du signe dit. Celuici a d'autant plus de prix qu'il a eu du mal à advenir à la parole. Aux ÉtatsUnis, il est de bon ton dans certains « talk shows » de venir raconter un traumatisme d'enfance, enfoui dans la mémoire et qui nous est revenu, récemment. Pourrait-on imaginer quelqu'un qui vient se raconter, faire l'aveu de son malheur, briser un silence de trente ans, et mentir, c'est-àdire, ne pas dire ce qui s'est passé réellement ? Et pourtant cela pourrait être le cas. Le silence partiel ou encore total, silence à l'intérieur de soi, oubli de l'événement, ne peut en aucun cas garantir la véracité de la parole. Il existe des choses que l'on a tues, ou que l'on construit comme tues qui sont fausses. La performativité de l'acte ne lui donne pas valeur de vérité. Or, pourtant, toute notre pensée du silence et de la parole est construite sur cette logique du vrai. Comme le décrit Foucault. Obtenir de quelqu'un qui souffre d'une maladie mentale l'aveu qu'il est fou est une procédure très ancienne dans la thérapeutique traditionnelle. L'aveu de sa propre folie constitue d'une part la vérité et d'autre part délivre le sujet de sa propre folie. « Nous avons là, en fait, l'inverse de l'acte de langage performatif. L'affirmation détruit chez le sujet parlant la réalité qui a rendu cette même affirmation vraie. Sur quelle conception de la vérité du discours et de la subjectivité cette pratique singulière, et pourtant si courante, se fonde? » (tome 4, 169). Ce qu'il faut penser alors, c'est que 
notre pensée de l'aveu est fondée sur une destruction du vrai au moment même où il apparaît dans le langage. Je suis folle, dirais-je et le dire me délivre de ma folie. Même l'aveu du meurtre constitue un étrange acte de langage. Si je dis une phrase telle que : " j'ai tué un ami, qui me demandait de l'aider à mourir ", par mon aveu, je mets en scène une vérité, mais du même coup je me délivre de quelque chose. Je n'ai plus autant tué que si je ne l'avais pas avoué : je suis déjà réintégrée dans le social. Le descriptif de mon acte est désamorcé dans le passage du silence à la parole. L'aveu me permet de dissoudre la charge de l'acte que j'ai posé. Peut-être parce que l'on présuppose que le passage du silence à la parole me coûte quelque chose, ne serait-ce que la possibilité de devoir me soumettre aux conséquences juridiques de ma parole.

Pour bien comprendre ce mécanisme du silence comme ce qui donne un prix à la parole, le mécanisme d'un silence comme difficile à rompre, on peut penser au cas de ce médecin affecté au camp d'Auschwitz, qui disait en 1998 au journal allemand Spiegel, en racontant ses crimes : "Je sais que je peux aller en prison pour ce que je vous raconte, mais je suis vieux. Je suis sûr que rien ne peut $\mathrm{m}^{\prime}$ arriver dans les deux ou trois ans. Et après, il sera trop tard ». Là, il s'agit de produire une parole que le juridique ne peut atteindre immédiatement. La prise de parole ne coûte rien à cet homme. Et en ce sens, elle ne lui permet pas de placer son aveu dans une économie sociale. Elle est suspension de la loi juridique et devient pure perversion, le comble du nazisme. Ici, l'aveu refait le crime, parce que le silence brisé ne met pas le sujet sous le coup de la loi ou encore de la punition possible.

Imaginons donc des scènes d'aveu, de bris de silence, qui mettent en scène la vérité, mais qui peut-être ne la disent pas. Des scènes d'aveu qui sont potentiellement de purs mensonges, des scènes d'aveu où le passage du silence à la parole ne donne aucune authenticité, ne coûte rien au sujet. Est-ce possible?

\section{L'aveu numéro deux. Mon parjure}

Lorsque j'avais 17 ans, je fus convoquée à la cour, afin de témoigner dans un procès qui avait lieu et où mon père, Iannis Mavrikakis, était l'accusé. Je suppliai mon père et son avocat de ne pas m'envoyer un appel à la comparution. Ils ne m'écoutèrent pas. Je dus aller témoigner, sous peine d'emprisonnement, je dus dire ce que je pensais de mon père. Je mentis. Je fus parjure. Je ne dis pas ma haine, mon enfance gâchée, les suicides à répétitions, les cris, la folie. Je ne dis pas tout cela. Je répondis aux questions, et là je dis la vérité, toute la vérité, je le jure. Mais 
je ne dis pas l'horreur. On ne me la demandait pas. Je dis toute la vérité, et gardai le silence. Mon père fut d'ailleurs accusé et fit de la prison. Malgré mon témoignage silencieux sur lui.

\section{L'aveu numéro trois. La syphilis de Charles Gaston et de Flora}

Un jour, je devais être adolescente, ma mère avoua à mon frère et à moi, que ses deux parents à elle n'étaient pas morts de leur belle mort, mais bien plutôt de syphilis que mon grand-père Charles Gaston Marchand, né le 12 mai 1889 à Bémécourt dans l'Eure (France) et mort le 24 octobre 1952 à Villers Bocage contracta auprès d'une prostituée en Normandie, où il vivait à l'époque.

Sa femme Flora Marchand (née Touret, le 24 novembre 1999, à Sainte Marguerite de l'Autel dans l'Eure (France)) mourut le 21 novembre 1970 à Montréal (Canada) des suites de cette maladie que son mari, mon grand-père lui "refila ", comme on dit. Mes grands-parents sont enterrés dans la même fosse du cimetière de Villers-Bocage (France). Ma mère tenait à ne plus garder le silence.

Elle ne nous dit rien d'autre sur les liens qui unissaient Charles et Flora.

\section{L'aveu numéro quatre. Celui de la mort imminente}

Pierre A. Larocque, metteur en scène, m'écrivit une lettre quatre jours avant sa mort où il m'avouait sa maladie et la possibilité de sa mort imminente. Nous nous étions souvent parlé avant cette lettre, mais c'est seulement quelques jours avant sa mort qu'il m'écrivit :

"Je ne vous l'ai peut-être pas dit, mais je suis atteint du sida. Cela se manifeste dans mes poumons. Cela a même un nom pneumocystis carinii ".

C'est le dernier aveu qu'il me fit avant de tomber dans le silence de la mort.

\section{La politique sociale du silence}

Il faut penser ici à l'accusation portée contre les homosexuels à l'endroit d'un prétendu silence sur leur homosexualité. Sur ce silence-là, l'État n'a pas encore légiféré. Il pourra le faire très prochainement. De cela, on ne peut en douter. Un jour, quelqu'un portera plainte sur le tort que lui a fait un homosexuel "dans le placard", silencieux. Pour l'instant, si l'on est homosexuel, il est des plus convenable de l'annoncer, de le crier sur tous les toits. Cela enlèverait une part de " responsabilité », dirais-je ironiquement. Et à ce jeu-là, on est déjà moins homosexuel, selon la logique de l'aveu. Ce n'est pas que l'aveu puisse changer l'orientation sexuelle du sujet, mais l'aveu va rendre possible l'intégration de la « différence » dans 
la parole sociale, va permettre de désamorcer la force possible du non-dit. Mais cette parole, bien sûr, coûtera au sujet. Elle ne lui sera pas facile. Briser les silences, dire, " en parler », voilà qui montre assez que la parole arrachée à soi-même est devenue salvatrice, et le silence condamnable. Foucault, théoricien de l'aveu s'il en fut, est souvent montré du doigt comme celui qui n'a pas assez avoué son homosexualité et qui n'a pas dit son sida, dont il connaissait peut-être l'existence. Le bon homosexuel, de nos jours, serait celui qui n'a plus de signe secret, celui qui aurait pu garder le silence, mais qui a fait le sacrifice personnel et social de s'exposer, de dire. Le prétendu silence de Foucault sur son homosexualité (« il ne l'a pas assez dit ») ou encore sur son sida montre assez combien le social demande la rupture du silence de l'homosexuel, et son intégration dans un code d'aveu.

Néanmoins, cela ne veut pas dire que la société actuelle demande la transparence en ce qui concerne les signes de $\mathrm{l}^{\prime}$ intimité ou encore une la définition de l'espace privé et public.

Je vais citer Adorno ici qui explique le silence auquel nous nous attendons collectivement :

Ainsi en est-il du fait de demander à quelqu'un des nouvelles de sa santé : comme notre éducation ne nous demande plus de poser cette question personne n'attend qu'elle soit posée, elle prend le sens d'une indiscrétion inquisitrice ou d'une offense. De même le silence qu'on observe sur certains sujets délicats devient indifférence vide dès lors qu'il n'y a pas de règle définissant ce dont on peut parler. Aussi les individus commencent-ils non sans raison, à réagir au tact avec hostilité : c'est ainsi qu'une certaine forme de politesse n'a pas tant pour effet qu'ils ont l'impression qu'on leur parle comme à des êtres humains qu'elle ne leur donne à soupçonner la situation inhumaine dans laquelle ils se trouvent. (32)

Je travaille sur le sida. Beaucoup de gens passent leur temps à me dire que je n'ai aucune légitimité à parler de cela. Si au moins je parlais du sida et des femmes, mais en général, je parle du sida et des homosexuels hommes. Je n'ai donc aucune légitimité à parler, parce que je ne suis pas un homme gai malade. On me dit parfois de travailler sur des choses bien à moi, sur des sujets communs à toutes les femmes, ou encore aux lesbiennes, sur le cancer du sein, par exemple. Mais, personne ne m'a 
demandé si j'étais malade, si je suis ou non séropositive. Tout le monde croit savoir que je n'ai pas le sida. C'est étrange. On ne me demande jamais des nouvelles de ma santé.

Je fais partie d'un comité universitaire qui a préparé un cours sur le sida : j'ai assisté à des dizaines de confessions de séropositivité, de bris de silence. Il est donc possible de donner des nouvelles de sa santé. Mais dans certaines conditions. L'éthique veut que jamais on ne demandera durant le cours aux étudiants s'ils sont ou non malades. Ce n'est pas qu'on ne veuille pas le savoir, parce que les professeurs et les assistants passent leur année à chercher, inconsciemment, et dans le fantasme le plus total, qui est séropositif ou non dans la classe, mais c'est qu' on ne veut pas l'entendre à moins qu'un dispositif psychologique ne soit mis en place.

On peut parler de sa séropositivité, mais de façon institutionnelle, en se déclarant séropositif, en en passant par l'aveu et la « psychologisation » à outrance. La maladie n'est plus privée, elle est codée, jusque dans son énonciation. Et la violence serait de faire entrer une certaine sorte de privé non codé à l'université. On croit qu'on fait exister quelque chose de ce genre en demandant à des séropositifs connus de venir parler, mais le discours de la séropositivité est extrêmement construit d'avance. Ce qui est presque un bien : là où il n'y aurait plus code, il n'y aurait plus rien du tout, plus de parole, puisque personne ne serait prêt à la violence d'une parole autre.

Pourquoi je travaille sur le sida ? Quelle est la bonne légitimité pour parler de la maladie ? Pourquoi devrais-je briser le silence ? Ou encore pourquoi devrais-je mettre en place un dispositif de bris de silence afin de prendre la parole ? On aimerait que je légitime ma parole dès que je produis du discours, que je passe aux aveux.

La violence de la rupture du silence sur la maladie n'aura pas lieu dans l'institution, et je pense que, même avec la meilleure volonté que nous avons dans certaines universités, où nous donnons, en sciences humaines, des cours sur le sida, l'université, comme institution, continue à se fantasmer comme un corps non malade qui ouvre ses portes de temps à autre à la maladie, à la parole de façon codée. Un corps qui ne laisse parler de la maladie et de la mort qu'à travers les spécialistes ou les malades, dans un discours social en partie prêt d'avance, dans du préfabriqué institutionnel. Qui a le droit de parler sur la maladie, de la maladie ? Qui a le droit de briser le silence ou de le garder ? Les politiques du dire qui sont à l'œuvre dans le discours de nos jours désamorcent toute la violence d'une prise de 
parole et en instituent une autre. Car il y a une violence à faire parler un sidéen du sida, comme il y en a une à le laisser dans le silence, puisque le silence et la parole sont désormais sous le coup de la loi de l'État. Il y a donc une violence à laisser dire ce qu'on attend, de faire taire ce que l'on ne saurait admettre et de ne plus pouvoir entendre aucune dissidence.

Quand les militants d'Act Up vont à la télévision pendant un téléthon, crachent sur les médias et empêchent les ministres de parler, les gens qui regardent ce téléthon sur le sida ne se font plus aussi généreux. Act Up serait trop violent. Il y a le discours sur la maladie qu'on aime et celui qu'on n'aime pas. Les silences qu'on désire et ceux qu'on trouve inacceptables. Les sidéens, on les veut la larme à l'œil, dans la proximité de leur mort ou livrant un beau combat. Dans un silence contrit, dans une parole qui les sauve.

Cataloguer les lieux de la prise de parole sur la maladie, forcer les lieux de parole pour mieux les cloisonner, voilà où la dialectique du silence et de la parole nous maintient à l'heure actuelle. Le silence est pris dans une politique du dire à laquelle il est presque impossible d'échapper. Si ce n'est encore que par un silence imposant.

Il faut peut-être penser à l'étoile jaune que l'on demandait aux Juifs de porter, ou encore au triangle rose qui était obligatoire pour les homosexuels durant une certaine partie du nazisme. Aucun silence n'était alors possible sur certains sujets. L'on était tenu à montrer tout le temps ces " je suis juive ", " je suis homosexuelle». Si le silence n'était pas possible alors, la parole non plus. Il n'y avait pas de sujet pour dire sa race ou son orientation sexuelle. C'est la loi, enfermée dans l'étoile ou le triangle qui le disait pour le sujet. Le silence doit nécessairement en passer par une parole qui se signe, qui continue par sa signature, d'échapper encore et malgré tout, aux signes préfabriqués de l'identité et de la vérité dévoilées.

\section{L'aveu numéro cinq. Celui de mon silence}

À travers tous mes aveux, mon amour, je n'ai pas réussi à dire. Je ne sais pas dire. Il n'y a que le psy, qui sache parler, et jamais elle ne m'adresse la parole. Je ne parle plus. Tu m'entends?

\section{Ouvrages cités}

Adorno, T. W. Minima Moralia. Payot, 1991.

Angot. C. L'inceste. Stock, 1999.

Foucault, M. Dits et écrits. 4 tomes. 1994. 